- Community Engagement

- Communications

Conclusion Promoting collaboration and supporting relationships between early career professionals is key within the adolescent healthcare field. Facilitating mentorship between the IAAH Young Professionals Network and IAAH Council fosters knowledge dissemination and leadership opportunities. The IAAH Young Professionals Network encourages applicants from diverse backgrounds, including, but not limited to: students, trainees, early career professionals (including health care providers, researchers, public health practitioners, advocates, scientists, social workers, pharmacists, nutritionists, health allies) who have a strong interest and/or experience in the adolescent health field. To the best of the authors knowledge, the IAAH-YPN network is the first global, inter- disciplinary global adolescent health initiative of its kind.

\section{P51 NUTRITIONAL INTERVENTIONS FOR ADOLESCENTS USING E-HEALTH TECHNOLOGIES (ICTS): A SYSTEMATIC REVIEW}

G Melo*, N Toral. Department of Nutrition, University of Brasilia, Brasilia, Brazil

\subsection{6/bmjpo-2019-RCPCH-SAHM.55}

Aims To identify e-health technologies and their main characteristics used for nutritional interventions for adolescents and to evaluate the quality and effectiveness of the studies.

Methods The full protocol is available on the PROSPERO website (\#CRD42016035882). A search was conducted across five databases (PubMed/MEDLINE, Scielo.ORG, Web of Science, PsycINFO, and Scopus) to identify papers describing nutritional interventions that used ICTs designed mainly for healthy adolescents. Full and original papers of randomized controlled trials, quasi-experimental or observational studies, published from 2005 to 2015, were included. The Effective Public Health Practice Project Quality Assessment Tool was used to assess study quality. Data was collected based on the guidance from the Centre for Reviews and Dissemination on undertaking reviews in healthcare

Results The search yielded 559 titles and abstracts. The number of studies which met the inclusion criteria was eleven. Recruitment of participants was mostly at schools. The followup of studies ranged from two weeks to two years. Interventional strategies included computer games, programs, text messages, and interactive CD-ROMs. More than $80 \%$ of studies (9 of 11) used computer-mediated Information and Communication Technologies. Five studies focused on multiple behaviours simultaneously. 6 interventions were developed based on a theoretical basis. Participants were exposed to interventions only once, daily, weekly, or according to a pre-determined number of lessons. Five studies had significant outcomes. All interventions that used games had significant outcomes. The quality assessment considered three studies as weak due to the non-representativeness of their samples and usage of non-validated questionnaires.

Conclusion Besides the heterogeneity and poor quality of the analyzed studies, it can be suggested that long-term interventions for adolescents that make use of frequent exposure to technological resources, and that have a theoretical component aimed at a single health behaviour change, tend to be more successful. Games showed to be a promising e-health platform for health education with adolescents.

\section{P52 MIGRANT YOUTH AND THE IMPACT OF CULTURAL VALUES, SOCIAL NETWORKS, ACCULTURATION AND CONDOM USE INTENTION AND BEHAVIOUR}

${ }^{1}$ RR Titus, ${ }^{2} \mathrm{JL}$ John-Langba*. 'Department of Social Development, University of Cape Town, Cape Town, South Africa; ${ }^{2}$ School of Applied Human Science, University of Kwazulu Natal, Kwazulu Natal, South Africa

\subsection{6/bmjpo-2019-RCPCH-SAHM.56}

Backgound Migrant health and adolescent well-being have become the focus point in current HIV intervention discourses as this particular cohort has an increased vulnerability to STIs such as HIV. As consistent use of condoms has a protective measure against STIs it is important to understand the key determinants that impact on their risky behaivours. In addition, it evaluates the extend that socio-cultural values and acculturation can predict condom use intentions among African youth residing in South Africa.

Methods Qualitative methodological approach was utilised with purposive and snowballing sampling technicques to explore migrant youth's intentions and behaviour towards condom use within their socio-cultural contexts. The study also measures their rate of acculturation and assimiliation within the current youth culture in South Africa regarding sexual risky behaviour and their psychology around condom use intentions and behaviour. A sample were drawn from Sub-Saharan African desent who migrate to South Africa. The mean age of respondents were 23 years rangin from 20 years to 25 years, with an equal gender distribution.

Results The results indicated that young migrants have a good knowledge of condom use and dual protection against pregancies and sexual transmitted infections. However, the use of condoms, even when freely available, is a contested issue as cultural values and traditional social networks have an impact on their intentions and behaivour towards condom use. Traditional gendered norms in sexual relationships and gendered expectations of condom use are current issues that migrant youth are grappling with. The nexus between cultural values and safer sexual choices places young migrants at risk as they are currently outside of the realms of socio-cultural contexts, with highter education expectations and delay of marriage customs.

Conclusion The study provide more insight into the current realities of transitioning young migrants who live outside of the boundaries of current socio-cultural paradigms.

\section{P53 SEX, BODY IMAGE AND RELATIONSHIPS: YOUNG PEOPLE WITH CANCER INFORMATION AND SUPPORT PREFERENCES}

${ }^{1}$ A Martins", ${ }^{1}$ RM Taylor, ${ }^{2}$ B Lobel, ${ }^{3} B$ McCan, ${ }^{3}$ L Soanes, ${ }^{1} J S$ Whelan, ${ }^{4}$ LA Fern. ${ }^{1}$ Cancer Division, University College London Hospitals NHS FT, London, UKi ${ }^{2}$ Research Department, Royal Central School of Speech and Drama University of London, London, UK; ${ }^{3}$ Children and Young People's Cancer Services, University College London Hospitals NHS FT, London, UK ${ }^{4}$ Young Adult and Germ Cell Clinical Studies Group, National Cancer Research Institute, London, UK

\subsection{6/bmjpo-2019-RCPCH-SAHM.57}

Aims Adolescents and young adults (AYA) diagnosed with cancer, broadly those aged 16-29, are known to have distinctive psychosocial and medical needs related to age and transitioning through significant life milestones at the time of diagnosis/ 